\title{
Cytokine Profile and Immunoglobulin E-mediated Serological Food Hypersensitivity in Patients With Irritable Bowel Syndrome With Diarrhea
}

\author{
Ryo Katsumata, ${ }^{1 *}$ Manabu Ishii, ${ }^{1}$ Suni Lee, ${ }^{2}$ Yukiko Handa, ${ }^{3}$ Takahisa Murao, ${ }^{1}$ Minoru Fujita, ${ }^{1}$ Hiroshi Matsumoto, ${ }^{1}$ Takemi Otsuki, ${ }^{2}$ \\ and Akiko Shiotani ${ }^{1}$ \\ ${ }^{\text {I} D i v i s i o n}$ of Gastroenterology, Kawasaki Medical School, Kurashiki, Okayama, Japan; ${ }^{2}$ Department of Hygiene, Kawasaki Medical School, \\ Kurashiki, Okayama, Japan; and ${ }^{3}$ Handa Clinic of Internal Medicine, Wakayama, Japan
}

\section{Background/Aims}

Food interaction, including food hypersensitivity, plays a key role in the pathogenesis of irritable bowel syndrome with diarrhea (IBS-D). Since only a few studies have been reported about the relationship between food hypersensitivity and IBS-D, we elucidate the prevalence of serological food hypersensitivity in patients with IBS-D and the characteristics of gastrointestinal symptoms and serum cytokine profiles in patients with IBS-D and serological food hypersensitivity.

\section{Methods}

Immunoglobulin E (Ig E)-mediated serological food hypersensitivity and serum cytokine levels were evaluated using the multiple allergen simultaneous test evaluating food allergen-specific serum lgE and Luminex Milliplex Panel containing multiple fluorescencelabeled beads. Class 2 or above was considered as IgE-mediated food hypersensitivity positive. The gastrointestinal symptom rating scale was used to evaluate symptoms.

\section{Results}

We enrolled 92 subjects, including 60 with IBS-D and 32 healthy controls. The percentages of patients with IgE-mediated serological food hypersensitivity were not significantly different between the groups (controls $=28.1 \%$ and IBS-D $=33.3 \%$ ). Serum IL- $1 \beta$, IL6, IL-8, macrophage inflammatory protein-1alpha, and TNF- $\alpha$ levels were higher in patients with IBS-D than in controls. Serum concentration of TNF- $\alpha$ (43.4 vs $21.8 \mathrm{pg} / \mathrm{mL}, P=0.009$ ) was higher in patients with IBS-D without IgE-mediated serological food hypersensitivity than those with food hypersensitivity.

\section{Conclusions}

One-third of Japanese patients with IBS-D showed IgE-mediated serological food hypersensitivity. The serum cytokine profile differed and was characterized by lower inflammatory cytokine levels in IBS-D with lgE-mediated serological food hypersensitivity. Serological test regarding IgE-mediated food hypersensitivity can detect a certain cluster of IBS-D.

(J Neurogastroenterol Motil 2018;24:415-421)

Key Words

Cytokines; Food hypersensitivity; Inflammation; Irritable bowel syndrome; Tumor necrosis factor-alpha

Received: September 18, 2017 Revised: November 26, 2017 Accepted: December 12, 2017

(.) This is an Open Access article distributed under the terms of the Creative Commons Attribution Non-Commercial License (http://creativecommons. org/licenses/by-nc/4.0) which permits unrestricted non-commercial use, distribution, and reproduction in any medium, provided the original work is properly cited.

*Correspondence: Ryo Katsumata, MD

Division of Gastroenterology, Kawasaki Medical School, 577 Matsushima, Kurashiki, Okayama 710-0192, Japan

Tel: +81-86-462-1111, Fax: +81-86-464-1195, E-mail: katsumata@med.kawasaki-m.ac.jp 


\section{Introduction}

Irritable bowel syndrome (IBS) is a common functional GI disorder characterized by altered bowel habit and abdominal pain without detectable organic causes on clinical examination. ${ }^{1}$ The pathogenesis of this heterogeneous disease has not been clearly understood, and several factors, such as psychosocial stress, alteration of serotonin signaling, low-grade inflammation, microbiota, barrier dysfunction on mucosal surfaces, and immune reaction to food, are thought to be related to this condition. ${ }^{2,3}$ Because the immune reaction to food by the intestinal mucosa is a crucial key in the pathogenesis, especially in patients with IBS-D, a relationship between IBS and food hypersensitivity currently has been researched. ${ }^{4,5}$ Several studies about immune mediated food hypersensitivity were reported so far. ${ }^{6,7}$ However, the prevalence of food hypersensitivity among IBS patients has not been investigated sufficiently, and its underlying mechanism remains unclear.

On the other hand, low-grade inflammation is also considered an important factor in the pathogenesis of IBS. ${ }^{8}$ Elevated serum inflammatory cytokines, such as IL-6, IL-8, and TNF- $\alpha$, and decreased serum anti-inflammatory cytokines, such as IL10, were detected in IBS patients compared with controls. ${ }^{9-11}$ This result represented a strong relationship between IBS and activated systemic inflammation, and in a study, the value of inflammatory cytokine was especially higher in patients with IBS-D among the 3 subtypes of IBS. Further, An et al, ${ }^{12}$ reported the upregulated expression of inducible nitric oxide and IL-1 $\beta$, which are closely associated with inflammation in patients with IBS-D compared with healthy controls. Despite the growing evidence of local and systemic inflammation in patients with IBS-D, to our knowledge, there is no published report evaluating the cytokine profile of patients with IBS-D and Immunoglobulin E (Ig E)-mediated serological food hypersensitivity.

We examined the prevalence of serological food hypersensitivity in patients with IBS-D compared with controls and evaluated gastrointestinal (GI) symptoms and cytokine profiles associated with IgE-mediated serological food hypersensitivity.

\section{Materials and Methods}

This cross-sectional study in Japan was approved by the Research Ethics Committee of Kawasaki Medical School and Hospital (IRB No. 2667), and informed consent in writing was obtained from each subject.

\section{Participants}

IBS-D was diagnosed in outpatients at Kawasaki Medical School Hospital using the Rome III $^{13}$ and Rome IV criteria. ${ }^{14}$ Control subjects were enrolled from those undergoing routine medical checkups who did not receive any medication or complained of any GI symptoms. The exclusion criteria were the following: age $<18$ years old; subjects with self-reported food allergy; allergic disease, including asthma and atopic dermatitis; systemic inflammatory disease; treatment of malignancy; and abdominal surgery. Subjects with a history of the aforementioned conditions within 10 years were also excluded. Colonoscopy was performed in all patients with IBS-D to confirm no presence of organic disorders. Appropriate sample size was calculated as 90 (controls $=30$ and IBS-D $=60$ ) in reference to past studies. ${ }^{6,15}$ We set $\alpha$ as 0.05 and $1-\beta$ as 0.808 .

\section{Serum Cytokines and Serological Food Hypersensitivity}

Peripheral venous blood samples obtained from each subject in the fasting state in the morning were stored at $-80^{\circ} \mathrm{C}$ until analysis. A multiple allergen simultaneous test was performed to detect food hypersensitivity. We used a multiple allergen simultaneous test assay (Hitachi Chemical Diagnostics, Mountain View, CA, USA) containing 20 kinds of food antigens, such as wheat, milk, egg, buckwheat, shrimp, crab, soybean, and more. This assay can measure several allergen-specific IgE simultaneously in each serum, ${ }^{16}$ and the sensitivity and specificity were $85 \%$ and $82 \%$, respectively. The results of this assay are represented as lumi-count (LC) and class (class $0=0-1.39$, class $1=1.40-2.77$, class $2=2.78-13.4$, class $3=13.5-58.0$, class $4=58.1-119$, class $5=120-150$, and class $6=160$ or above). Class 2 or above was judged as positive for IgE-mediated serological food hypersensitivity. ${ }^{18} \mathrm{~A}$ subject who had at least one positive allergen was considered to be positive for IgE-mediated serological food hypersensitivity. Serum cytokine levels were measured by a Luminex Milliplex MAP Human Cytokine/Chemokine Magnetic Bead Panel (HCYMAG-60KPX29; Merck Millipore, Billerica, MA, USA). We investigated 13 serum cytokines in reference to prior studies on IBS and allergic disease. $^{9-11,19}$ The cytokines, including macrophage inflammatory protein (MIP)-1 $\alpha$, IFN- $\gamma$, eotaxin, IL-1 $\beta$, IL-4, IL-5, IL-6. IL8, IL-10, IL-12p70, IL-13, IL-17, and TNF- $\alpha$, were evaluated using fluorescence-labeled beads. The descriptions of each cytokine are listed in Supplementary Table 1. As described previously, the beads were incubated with the serums overnight at $4^{\circ} \mathrm{C}$. They were incubated with streptavidin-phycoerythrin after incubation with 
detection antibodies at room temperature for 1 hour. Lastly, plate reading and analysis were performed using Luminex 200 xPOTENT System version 3.1 (EMD Millipore). ${ }^{20}$

\section{Questionnaire}

GI symptoms were assessed using the GI symptom rating scale (GSRS) to compare patients with and without serological food hypersensitivity. The 15 questions on the GSRS related to 5 major GI symptoms (reflux, abdominal pain, indigestion, diarrhea, and constipation) and were scored on a scale of $1-7 .^{21}$

\section{Statistical Methods}

Continuous and normally distributed variables were expressed as mean and standard deviation. Categorical, non-normally distributed continuous variables or frequencies were expressed as median, interquartile range, and percentage. Normal distribution and homoscedasticity were evaluated with the Shapiro-Wilk and Levene tests. Spearman test was used to investigate correlations between GI symptoms and serum cytokine levels. Student's t test (continuous variables), the Mann-Whitney $U$ test (categorical variables or non-normally distributed continuous variables), or the $\chi^{2}$ test (frequencies) was used to compare the variables between 2 groups. Two-sided $P$-values less than 0.05 were considered statistically significant. Statistical analyses were calculated by SPSS statistical software (version 24.0; IBM Corp, Armonk, NY, USA) and IBM SPSS Bootstrapping (version 24.0; IBM Corp) to obtain 1000 random samples to derive estimates of their $95 \%$ confidence intervals.

\section{Results}

The 92 subjects ( 44 men and 48 women) enrolled in this study consisted of 60 with IBS-D and 32 controls. All demographic data, such as age, sex, and body mass index, were not significantly different between the controls and patients with IBS-D (Table 1). The prevalence of $\mathrm{IgE}$-mediated serological food hypersensitivity in patients with IBS-D was $33.3 \%$, which was not significantly different compared to the controls $(28.1 \%, P=0.646)$. Summary of the results of all food allergens is shown in Table 2 . The most frequent foods with positive results in controls were buckwheat and shrimp (9.4\%), whereas wheat showed the highest positivity in patients with IBS-D (10.0\%). In patients with IBS-D, the median GSRS scores and scores obtained by Bootstrapping were not different between

Table 1. Comparison of Demographic Data, Prevalence of Immunoglobulin E-mediated Serological Food Hypersensitivity and Serum Cytokine Profile Between Patients With Irritable Bowel Syndrome With Diarrhea and Controls

\begin{tabular}{|c|c|c|c|}
\hline Variables & Control $(\mathrm{n}=32)$ & IBS-D $(n=60)$ & $P$-value \\
\hline Age (mean [SD], yr) & $42.5(11.2)$ & $47.9(20.5)$ & $0.169^{\mathrm{a}}$ \\
\hline Male sex (n [\%]) & $18(56.2)$ & $26(43.3)$ & $0.336^{\mathrm{b}}$ \\
\hline $\mathrm{BMI}\left(\right.$ mean $\left.[\mathrm{SD}], \mathrm{kg} / \mathrm{m}^{2}\right)$ & $21.6(3.3)$ & $22.3(4.2)$ & $0.495^{\mathrm{a}}$ \\
\hline $\begin{array}{l}\text { IgE-mediated serological food } \\
\text { hypersensitivity positive (n }[\%])\end{array}$ & $9(28.1)$ & $20(33.3)$ & $0.646^{\mathrm{b}}$ \\
\hline \multicolumn{4}{|c|}{ Serum cytokine (median [IQR], pg/mL) } \\
\hline IL-1 $\beta$ & $0.98(0.59-1.93)$ & $394.1(57.6-480.0)$ & $0.004^{\mathrm{c}}$ \\
\hline IFN- $\gamma$ & $9.7(3.9-27.2)$ & $13.1(7.2-27.0)$ & $0.222^{c}$ \\
\hline MIP-1 $\alpha$ & $27.3(24.5-29.7)$ & $197.8(42.9-698.4)$ & $<0.001^{\mathrm{c}}$ \\
\hline Eotaxin & $122.4(104.4-139.1)$ & $147.3(121.1-200.9)$ & $0.051^{\mathrm{c}}$ \\
\hline IL-6 & $7.1(1.1-11.9)$ & $22.7(4.3-60.2)$ & $<0.001^{\mathrm{c}}$ \\
\hline IL-8 & $8.3(6.6-15.9)$ & $408.7(86.9-1119.5)$ & $<0.001^{\mathrm{c}}$ \\
\hline IL-10 & $2.8(1.2-5.9)$ & $4.2(2.1-9.9)$ & $0.106^{\mathrm{c}}$ \\
\hline IL-12p70 & $14.3(6.1-43.6)$ & $3.7(1.5-23.6)$ & $0.192^{\mathrm{c}}$ \\
\hline IL-13 & $118.9(8.6-217)$ & $20.7(8.3-89.4)$ & $0.324^{c}$ \\
\hline IL-17 & $4.1(1.0-21.7)$ & $4.5(2.7-11.8)$ & $0.397^{\mathrm{c}}$ \\
\hline TNF- $\alpha$ & $7.1(6.7-10.1)$ & $36.9(18.1-74.7)$ & $<0.001^{\mathrm{c}}$ \\
\hline
\end{tabular}

IBS-D, irritable bowel syndrome with diarrhea; SD, standard deviation; BMI, body mass index; IQR, interquartile range; IL-1 $\beta$, interleukin-1beta; IFN- $\gamma$, interferon-gamma; MIP-1 $\alpha$, macrophage inflammatory protein-1alpha; IL-6, interleukin-6; IL-8, interleukin-8; IL-12p70, interleukin-12p70; IL-13, interleukin-13; IL-17, interleukin-17; TNF- $\alpha$, tumor necrosis factor-alpha. The subject who has at least one food allergen with class 2 or above was considered as being positive for IgE-mediated serological food hypersensitivity.

$P$-value was calculated by the Student's ${ }^{a} t$ test, ${ }^{b}$ chi-squared test, and ${ }^{\mathrm{c}}$ Mann-Whitney $\mathrm{U}$ test. 
Table 2. Summary of Subjects With Positive Results Regarding All Food Allergens in Control and Irritable Bowel Syndrome With Diarrhea

\begin{tabular}{lcc}
\hline Food allergen & Control $(\mathrm{n}=32)$ & IBS-D $(\mathrm{n}=60)$ \\
\hline Wheat & $1(3.1)$ & $6(10.0)$ \\
Soybean & $1(3.1)$ & $4(6.7)$ \\
Rice & $1(3.1)$ & $3(5.0)$ \\
Tuna & $0(0.0)$ & $2(3.3)$ \\
Salmon & $0(0.0)$ & $1(1.7)$ \\
Shrimp & $3(9.4)$ & $3(5.0)$ \\
Crab & $1(3.1)$ & $3(5.0)$ \\
Milk & $0(0.0)$ & $1(1.7)$ \\
Beef & $0(0.0)$ & $1(1.7)$ \\
Chicken & $0(0.0)$ & $0(0.0)$ \\
Egg white & $0(0.0)$ & $4(6.7)$ \\
Buckwheat & $3(9.4)$ & $2(3.3)$ \\
Peanuts & $2(6.3)$ & $4(6.7)$ \\
Ovomucoid & $1(3.1)$ & $4(6.7)$ \\
Sesame & $1(3.1)$ & $6(10.0)$ \\
Kiwifruit & $0(0.0)$ & $3(5.0)$ \\
Banana & $0(0.0)$ & $4(6.7)$ \\
Pork & $0(0.0)$ & $2(3.3)$ \\
Peach & $1(3.1)$ & $1(1.7)$ \\
Tomato & $1(3.1)$ & $0(0.0)$ \\
\hline
\end{tabular}

IBS-D, irritable bowel syndrome with diarrhea.

Number of positive subjects and percentage were listed on each allergen.

A class 2 or above was judged as positive for Immunoglobulin E-mediated serological food hypersensitivity. The numbers includes duplication.

Table 3. Gastrointestinal Symptom Rating Scale Scores in Patients With Irritable Bowel Syndrome With Diarrhea

\begin{tabular}{lccc}
\hline \multirow{2}{*}{$\begin{array}{c}\text { Symptom score } \\
\text { (median }[\mathrm{IQR}])\end{array}$} & \multicolumn{2}{c}{$\begin{array}{c}\text { IgE-mediated serological } \\
\text { food hypersensitivity }\end{array}$} & \\
\cline { 2 - 3 } & $\begin{array}{c}\text { Negative } \\
(\mathrm{n}=40)\end{array}$ & $\begin{array}{c}\text { Positive } \\
(\mathrm{n}=20)\end{array}$ & \\
\hline Reflux & $1.7(1.3-2.7)$ & $2.0(1.7-2.7)$ & 0.574 \\
Abdominal pain & $2.9(2.3-4.6)$ & $2.0(1.7-3.9)$ & 0.610 \\
Indigestion & $2.1(1.6-2.9)$ & $2.3(1.8-2.5)$ & 0.894 \\
Diarrhea & $4.2(3.7-5.3)$ & $4.3(4.1-5.0)$ & 0.859 \\
Constipation & $2.1(1.7-2.9)$ & $2.0(1.7-2.3)$ & 0.445 \\
\hline
\end{tabular}

IQR, interquartile range.

The subject who has at least one food allergen with class 2 or above was considered as being positive for Immunoglobulin E ( $\mathrm{Ig} \mathrm{E}$ )-mediated serological food hypersensitivity.

$P$-value was calculated by Mann-Whitney $\mathrm{U}$ test.

patients with and without IgE-mediated serological food hypersensitivity according to each symptom (Table 3 and Supplementary Table 2).

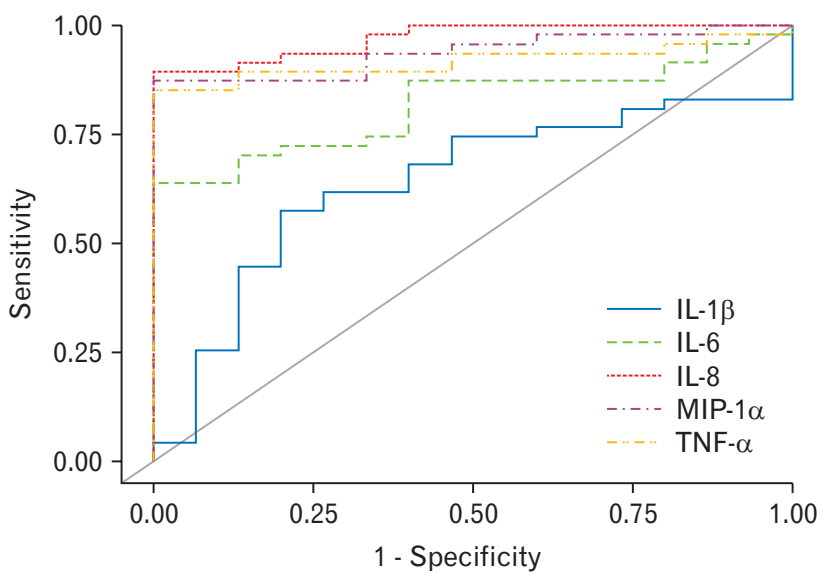

Figure 1. Receiver operating characteristic curves based on IL-1 $\beta$, macrophage inflammatory protein-1alpha (MIP-1 $\alpha$ ), IL-6, IL-8, and $\mathrm{TNF}-\alpha$ levels for irritable bowel syndrome with diarrhea versus healthy controls.

\section{Serum Cytokines}

Serum median IL-1 $\beta$, MIP-1 $\alpha$, IL-6, IL-8, and TNF- $\alpha$ levels in patients with IBS-D were significantly higher than those of controls (Table 1). The receiver operating characteristic (ROC) curves of the cytokine levels for IBS-D versus healthy controls are shown in Figure 1. Serum cytokine levels of IL-6, TNF- $\alpha$, MIP$1 \alpha$, and IL-8 provided adequate overall differentiation of IBS-D cases from healthy controls (area under the curve of IL- 6 , TNF- $\alpha$, MIP- $1 \alpha$, and IL-8 is $0.817,0.918,0.938$, and 0.970 , respectively). Serum cytokine levels were not significantly correlated with GI symptoms scores. All $r$ value and Figures are shown in Supplementary Table 3 and Supplementary Figure. Serum TNF- $\alpha$ (43.4 vs $21.8 \mathrm{pg} / \mathrm{mL}, P=0.009)$ concentration was significantly higher in patients without versus those with IgE-mediated serological food hypersensitivity (Table 4). The results after using Bootstrapping showed a similar tendency that the levels of inflammatory cytokines such as TNF- $\alpha$ were higher in patients without IgE-mediated serological food hypersensitivity (Supplementary Table 4). Among the 4 groups (controls and patients with and without serological food hypersensitivity), patients with IBS-D without serological food hypersensitivity showed the highest serum TNF- $\alpha$ titer (Fig. 2). IL-4 and IL-5 were detected only in less than half of the subjects (control group: IL-4 $=18.7 \%$, IL-5 $=37.5 \%$; IBS group: IL-4 $=16.7 \%$, IL-5 $=46.7 \%$; data not shown).

\section{Discussion}

We investigated the characteristics of serum cytokine profiles 
Table 4. Comparison of Serum Cytokine Profile Between Immunoglobulin E-mediated Serological Food Hypersensitivity Negative and Positive in Patients With Irritable Bowel Syndrome With Diarrhea

\begin{tabular}{lccc}
\hline \multirow{2}{*}{$\begin{array}{c}\text { Serum cytokine } \\
\text { level (median } \\
\text { IQR], pg/mL) }\end{array}$} & \multicolumn{2}{c}{$\begin{array}{c}\text { IgE-mediated serological food } \\
\text { hypersensitivity }\end{array}$} & \\
\cline { 2 - 3 } & Negative $(\mathrm{n}=40)$ & Positive $(\mathrm{n}=20)$ & \\
\hline IL-1 $\beta$ & $4.2(1.8-6.5)$ & $1.8(0.7-9.6)$ & 0.303 \\
IFN- $\gamma$ & $13.8(9.1-33.6)$ & $11.8(5.6-25.9)$ & 0.388 \\
MIP-1 $\alpha$ & $300.2(94.1-844.7)$ & $124.7(21.6-412.6)$ & 0.106 \\
Eotaxin & $163.8(126.7-198.9)$ & $134.9(94.8-211.6)$ & 0.495 \\
IL-6 & $32.4(8.1-74.5)$ & $7.5(2.6-40.9)$ & 0.083 \\
IL-8 & $468.7(65.2-1368.6)$ & $316.6(87.5-700.1)$ & 0.397 \\
IL-10 & $4.4(2.1-9.3)$ & $3.9(2.0-12.9)$ & 0.860 \\
IL-12p70 & $3.5(1.3-24.7)$ & $4.7(2.4-21.7)$ & 0.900 \\
IL-13 & $20.5(5.2-102.5)$ & $26.8(10.9-81.8)$ & 0.833 \\
IL-17 & $5.2(2.7-12.5)$ & $3.3(1.7-10.4)$ & 0.122 \\
TNF- $\alpha$ & $43.4(24.7-88.4)$ & $21.8(9.4-48.3)$ & 0.009 \\
\hline
\end{tabular}

IQR, interquartile range; MIP-1 $\alpha$, macrophage inflammatory protein-1alpha. The subject who has at least one food allergen with class 2 or above was considered as being positive for Immunoglobulin E-mediated serological food hypersensitivity.

$P$-value was calculated by the Mann-Whitney $\mathrm{U}$ test.

in patients with IBS-D and sex- and age-matched healthy subjects related to serological food hypersensitivity. Similar to past studies, ${ }^{9-11}$ we detected a difference in several cytokine levels of IL-1 $\beta$, MIP$1 \alpha$, IL-6, IL-8, and TNF- $\alpha$ between controls and patients with IBS-D. MIP-1 is a member of the CC subfamily of chemokines that has 2 adjacent cysteines in their amino acid sequence. ${ }^{22}$ They were initially detected in $1988{ }^{23}$ and later, they were shown to be 2 proteins, termed MIP- $1 \alpha$ and MIP-1 $1 .^{24}$ MIP- $1 \alpha$ was shown to modulate the activity of neutrophils and immune inflammation. ${ }^{25}$ These inflammatory cytokines are connected strongly to the pathogenesis of IBS, particularly in IBS-D, and elevated serum concentrations of these molecules seem to be consistent with past reports.

Furthermore, we revealed the difference between the subgroup of patients with IBS-D with and without IgE-mediated serological food hypersensitivity. Median inflammatory cytokine levels were highest in patients without serological food hypersensitivity and decreased in order in patients with, controls without, and controls with serological food hypersensitivity. The cytokine profiles of patients without serological food hypersensitivity were similar to those of patients with IBS-D in past reports. ${ }^{9-11}$ TNF- $\alpha$ is a well-known cytokine that induces inflammation. Elevation of this cytokine has been confirmed in several types of inflammatory disease, such as inflammatory bowel disease, rheumatoid arthritis, and psoriasis. ${ }^{26}$ Elevation of TNF- $\alpha$ can promote the release of the corticotrophin-

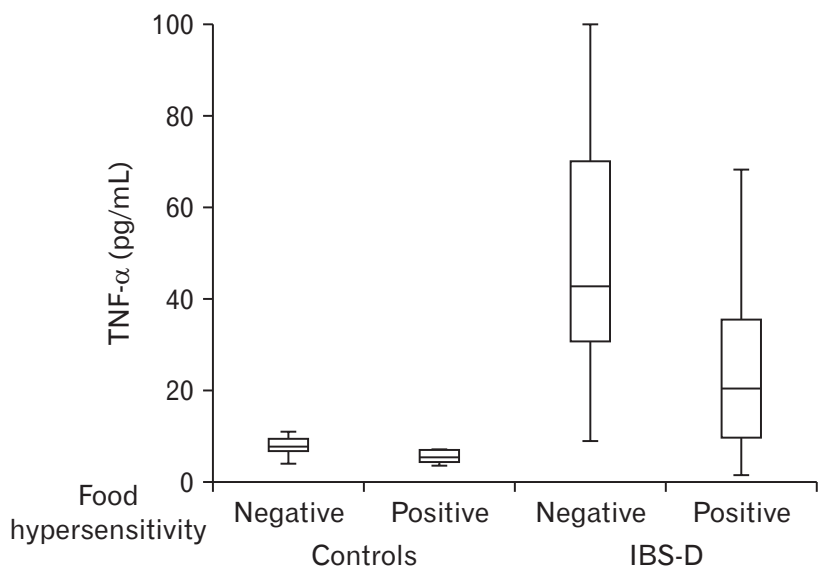

Figure 2. Comparison of cytokine concentration of TNF- $\alpha$ among 4 groups (controls with and without serological food hypersensitivity and irritable bowel syndrome with diarrhea [IBS-D] patients with and without serological food hypersensitivity).

releasing hormone in the brain, which results in GI symptoms. ${ }^{27,28}$ In addition, TNF- $\alpha$ functions to increase intestinal mucosal permeability. ${ }^{29}$ The functions can be associated with the pathogenesis of IBS-D, and in our result of ROC curve, TNF- $\alpha$ levels were reliable to differentiate patients with IBS-D from healthy subjects. Therefore, the fact that patients with IBS-D without IgE-mediated serological food hypersensitivity showed higher levels of TNF- $\alpha$ than patients with IBS-D without IgE-mediated serological food hypersensitivity were more plausible as conventional IBS-D than those with.

One-third of Japanese subjects showed serological food hypersensitivity. The prevalence of serological food hypersensitivity was not different between Japanese patients with IBS-D and healthy controls. So far, little has been reported about the relationship between serological food hypersensitivity and IBS. ${ }^{30}$ Although a higher prevalence of IBS was detected among patients with selfreported food hypersensitivity, ${ }^{31}$ there was no evidence that those with IBS had a greater prevalence of serological food hypersensitivity compared to healthy controls. An almost equal prevalence of food tolerance was reported among patients with IBS compared to healthy controls. ${ }^{32}$ We confirmed that our result in this study was also consistent with prior reports in other countries.

Interestingly, despite the different patterns on the cytokine profile, GI symptom scores were similar in patients with and without serological food hypersensitivity. Food allergy can cause many GI symptoms, including diarrhea and abdominal pain, observed in patients with IBS-D ${ }^{33}$ indicating that such patients diagnosed according to symptoms might include the patient cluster that is influenced 
by serological food hypersensitivity. Since they can show a similar extent of abdominal symptoms, detection of the cluster only according to symptoms seems to be difficult.

Taking our results for granted, a feasible option might be to eliminate specific foods detected by a serological test and to prescribe an antiallergic drug for patients with serological food hypersensitivity. Indeed, past studies have proved the efficacy of food elimination for patients whose IBS was diagnosed by a blood IgG test. ${ }^{34} \mathrm{~A}$ mast cell stabilizer used to treat allergic disease, such as asthma and allergic conjunctivitis, has been reported to improve symptoms and increase the threshold for discomfort in a doubleblind randomized study. ${ }^{35}$ Another case report indicated the effectiveness of antiallergic drugs, such as immunoglobulin $\mathrm{E}$ ( $\mathrm{IgE}$ ) antibody, in a patient with IBS with mixed bowel habits. ${ }^{36}$ This study suggested that serological food hypersensitivity test can detect a cluster with IgE-mediated serological food hypersensitivity and lower systemic inflammation. Considering past studies, the cluster might be susceptible for food elimination and antiallergic drugs.

The limitations of this study included the small number of subjects, and selection bias might affect our results. Further studies with increased number of subjects are required to confirm our findings, particularly those concerning the difference in the cytokine profile between food hypersensitivity positive and negative subjects. However, some significant differences were discovered in this research. These data would be meaningful in understanding the pathogenesis of IBS-D and in designing further research, including a prospective interventional study. Another limitation is the process used to diagnose patients with serological food hypersensitivity. Food allergy is diagnosed basically by following guidelines in clinical practice. ${ }^{37}$ This process begins with a self-reported history of allergic symptoms, such as skin trouble. However, a discrepancy was reported in the prevalence of food allergy between patients who self-reported and those who were serologically positive for food hypersensitivity. ${ }^{38}$ Because of these data, we used objective serological methods to diagnose food hypersensitivity. We assessed only 20 types of food hypersensitivity using the IgE test and did not evaluate antigens other than those 20 and antigen-specific $\operatorname{IgG}$, which implies a biomarker for delayed allergy and food intolerance. ${ }^{39}$ However IgE-mediated food hypersensitivity is caused mainly by particular antigens evaluated in our research, ${ }^{30}$ and basically, food allergies are caused through immediate-allergy, which is evaluated using the IgE test. The target of antiallergy drugs, such as an $\mathrm{H} 1$ blocker, and $\mathrm{IgE}$ antibody is IgE-mediated reaction. Thus, our methods that evaluate antigen-specific $\mathrm{IgE}$ reaction including 20 types of food were feasible to choose candidates who received food elimination and antiallergy drug therapy.

In summary, the prevalence of IgE-mediated serological food hypersensitivity in patients with IBS-D was not significantly different compared to healthy controls and the cytokine profile was different depending on serological food hypersensitivity in patients with IBS-D. Patients with IgE-mediated serological food hypersensitivity showed lower serum inflammatory cytokine levels than those without. Our results indicated that a serological test for food hypersensitivity would be the option to detect a specific cluster among patients with IBS-D. Further researches including prospective studies are expected following these results.

Acknowledgements: The authors thank Ms Shoko Yamamoto, Aiko Mizukawa and Miho Miyata for their technical help.

Financial support: Grant support from Kawasaki Medical School Project Grant (29-B-039).

\section{Conflicts of interest: None.}

Author contributions: Ryo Katsumata contributed to the study design, performed the statistical analyses, and wrote the manuscript; Manabu Ishii designed the study protocol and mainly collected the blood samples; Suni Lee and Takemi Otsuki assessed serum cytokine; Yukiko Handa, Takahisa Murao, and Minoru Fujita collected blood samples and clinical data; Hiroshi Matsumoto helped in analyzing data; and Akiko Shiotani contributed to the study concept and provided a revision of the manuscript.

\section{References}

1. Enck P, Aziz Q, Barbara G, et al. Irritable bowel syndrome. Nat Rev Dis Primers 2016;2:16014.

2. Drossman DA. Functional Gastrointestinal disorders: history, pathophysiology, clinical features and Rome IV. Gastroenterology 2016;150:12621279, e2.

3. Saha L. Irritable bowel syndrome: pathogenesis, diagnosis, treatment, and evidence-based medicine. World J Gastroenterol 2014;20:67596773.

4. Hayes PA, Fraher MH, Quigley EM. Irritable bowel syndrome: the role of food in pathogenesis and management. Gastroenterol Hepatol 2014;10:164-174.

5. Rizzi A, Nucera E, Laterza L, et al. Irritable bowel syndrome and nickel allergy: what is the role of the low nickel diet? J Neurogastroenterol Motil 2017;23:101-108.

6. Zuo XL, Li YQ, Li WJ, , et al. Alterations of food antigen-specific serum immunoglobulins $\mathrm{G}$ and $\mathrm{E}$ antibodies in patients with irritable bowel syndrome and functional dyspepsia. Clin Exp Allergy 2007;37:823-830. 
7. Ligaarden SC, Lydersen S, Farup PG. IgG and IgG4 antibodies in subjects with irritable bowel syndrome: a case control study in the general population. BMC Gastroenterol 2012;12:166.

8. Barbara G, Cremon C, Annese V, et al. Randomised controlled trial of mesalazine in IBS. Gut 2016;65:82-90.

9. Choghakhori R, Abbasnezhad A, Hasanvand A, Amani R. Inflammatory cytokines and oxidative stress biomarkers in irritable bowel syndrome: association with digestive symptoms and quality of life. Cytokine 2017;93:34-43.

10. Seyedmirzaee S, Hayatbakhsh MM, Ahmadi B, et al. Serum immune biomarkers in irritable bowel syndrome. Clin Res Hepatol Gastroenterol 2016;40:631-637.

11. Bashashati M, Rezaei N, Shafieyoun A, et al. Cytokine imbalance in irritable bowel syndrome: a systematic review and meta-analysis. Neurogastroenterol Motil 2014;26:1036-1048.

12. An S, Zong G, Wang Z, Shi J, Du H, Hu J. Expression of inducible nitric oxide synthase in mast cells contributes to the regulation of inflammatory cytokines in irritable bowel syndrome with diarrhea. Neurogastroenterol Motil 2016;28:1083-1093.

13. Longstreth GF, Thompson WG, Chey WD, Houghton LA, Mearin F, Spiller RC. Functional bowel disorders. Gastroenterology 2006;130:1480-1491.

14. Mearin F, Lacy BE, Chang L, et al. Bowel disorders. Gastroenterology 2016;150:1393-1407, e5.

15. Fleiss JL, Tytun A, Ury HK. A simple approximation for calculating sample sizes for comparing independent proportions. Biometrics 1980;36:343-346.

16. Miller SP, Marinkovich VA, Riege DH, et al. Application of the MAST immunodiagnostic system to the determination of allergen-specific IgE. Clin Chem 1984;30:1467-1472.

17. Han M, Shin S, Park H, Park KU, Park MH, Song EY. Comparison of three multiple allergen simultaneous tests: RIDA allergy screen, MAST optigen, and polycheck allergy. Biomed Res Int 2013;2013:340513.

18. Ishimura N, Furuta K, Sato S, Ishihara S, Kinoshita Y. Limited role of allergy testing in patients with eosinophilic gastrointestinal disorders. J Gastroenterol Hepatol 2013;28:1306-1313.

19. Johnston LK, Chien KB, Bryce PJ. The immunology of food allergy. J Immunol 2014;192:2529-2534.

20. Maki Y, Nishimura Y, Toyooka S, et al. The proliferative effects of asbestos-exposed peripheral blood mononuclear cells on mesothelial cells. Oncol Lett 2016;11:3308-3316.

21. Svedlund J, Sjödin I, Dotevall G. GSRS--a clinical rating scale for gastrointestinal symptoms in patients with irritable bowel syndrome and peptic ulcer disease. Dig Dis Sci 1988;33:129-134.

22. Zlotnik A, Yoshie O. Chemokines: a new classification system and their role in immunity. Immunity 2000;12:121-127.

23. Wolpe SD, Davatelis G, Sherry B, et al. Macrophages secrete a novel heparin-binding protein with inflammatory and neutrophil chemokinetic properties. J Exp Med 1988;167:570-581.
24. Sherry B, Tekamp-Olson P, Gallegos C, et al. Resolution of the two components of macrophage inflammatory protein 1 , and cloning and characterization of one of those components, macrophage inflammatory protein 1 beta. J Exp Med 1988;168:2251-2259.

25. Hsieh CH, Frink M, Hsieh YC, et al. The role of MIP-1 alpha in the development of systemic inflammatory response and organ injury following trauma hemorrhage. J Immunol 2008;181:2806-2812.

26. Kalliolias GD, Ivashkiv LB. TNF biology, pathogenic mechanisms and emerging therapeutic strategies. Nat Rev Rheumatol 2016;12:49-62.

27. Dinan TG, Quigley EM, Ahmed SM, et al. Hypothalamic-pituitarygut axis dysregulation in irritable bowel syndrome: plasma cytokines as a potential biomarker? Gastroenterology 2006;130:304-311.

28. Fukudo S, Nomura T, Hongo M. Impact of corticotropin-releasing hormone on gastrointestinal motility and adrenocorticotropic hormone in normal controls and patients with irritable bowel syndrome. Gut $1998 ; 42: 845-849$.

29. Ma TY, Iwamoto GK, Hoa NT, et al. TNF-alpha-induced increase in intestinal epithelial tight junction permeability requires NF-kappa B activation. Am J Physiol Gastrointest Liver Physiol 2004;286:367-376.

30. Mansueto P, D'Alcamo A, Seidita A, Carroccio A. Food allergy in irritable bowel syndrome: the case of non-celiac wheat sensitivity. World J Gastroenterol 2015;21:7089-7109.

31. Lillest $\varnothing 1$ K, Helgeland L, Arslan Lied G, et al. Indications of 'atopic bowel' in patients with self-reported food hypersensitivity. Aliment Pharmacol Ther 2010;31:1112-1122.

32. Karamanolis G, Tack J. Nutrition and motility disorders. Best Pract Res Clin Gastroenterol 2006;20:485-505.

33. Patel BY, Volcheck GW. Food allergy: common causes, diagnosis, and treatment. Mayo Clin Proc 2015;90:1411-1419.

34. Atkinson W, Sheldon TA, Shaath N, Whorwell PJ. Food elimination based on IgG antibodies in irritable bowel syndrome: a randomised controlled trial. Gut 2004;53:1459-1464.

35. Klooker TK, Braak B, Koopman KE, et al. The mast cell stabiliser ketotifen decreases visceral hypersensitivity and improves intestinal symptoms in patients with irritable bowel syndrome. Gut 2010;59:1213-1221.

36. Pearson JS, Niven RM, Meng J, Atarodi S, Whorwell PJ. Immunoglobulin $\mathrm{E}$ in irritable bowel syndrome: another target for treatment? A case report and literature review. Therap Adv Gastroenterol 2015;8:270277.

37. Ebisawa M. Management of food allergy in Japan "food allergy management guideline 2008 (revision from 2005)" and "guidelines for the treatment of allergic diseases in schools". Allergol Int 2009;58:475-483.

38. Muraro A, Werfel T, Hoffmann-Sommergruber K, et al. EAACI food allergy and anaphylaxis guidelines: diagnosis and management of food allergy. Allergy 2014;69:1008-1025.

39. Shakoor Z, AlFaifi A, AlAmro B, AlTawil LN, AlOhaly RY. Prevalence of IgG-mediated food intolerance among patients with allergic symptoms. Ann Saudi Med 2016;36:386-390. 